\title{
GLOBAL DIMENSION OF ARTINIAN RINGS ${ }^{1}$
}

\section{ABRAHAM ZAKS}

Introduction. It was shown by Auslander in [1] that in an Artinian ring $R$ with radical $N \neq 0$ there exists an indecomposable left ideal $I$ of square zero that satisfies the equality l.p.dim $I=1 . p \cdot \operatorname{dim} N$.

Considering only the cyclic left ideals in an Artinian ring $R$ we obtain a bound for the global dimension of $R$ from Sup 1.p.dim $J$ (where $J$ ranges over all cyclic left ideals for which $J^{2}=0, J N=0$, and $\operatorname{Hom}_{R}(J, J)$ is a division ring) and from the length of the radical.

The author wishes to thank Professors M. Auslander and M. I. Rosen for their helpful remarks and suggestions in the preparation of this paper.

1. Global dimension in Artinian rings. For the rest we assume that all rings have an identity, all the ideals are left ideals, and all modules are unitary left modules, unless otherwise specified.

For a left $R$-module $M$ we set $M^{*}=\operatorname{Hom}_{R}(M, R)$, and we write $l(M)$ for its length.

Consider the map $\mu: M^{*} \otimes_{R} M \rightarrow \operatorname{Hom}_{R}(M, M)$ defined by $\mu\left(f \otimes m_{1}\right)$ $\left(m_{2}\right)=f\left(m_{2}\right) \cdot m_{1}$ for $f \in M^{*}$ and $m_{1}, m_{2} \in M$. It is well known that $\operatorname{Im} \mu$ is a two-sided ideal in $\operatorname{Hom}_{R}(M, M)$. Furthermore, $M$ is a finitely generated projective $R$-module iff $\mu$ is an epimorphism, in which case it is an isomorphism (e.g. [2, appendix]).

The following are two useful lemmas.

Lemma 1. Let $J$ be an ideal in an arbitrary ring. If $J^{2} \neq 0$ and $\operatorname{Hom}_{R}(J, J)$ is a simple ring, then $J$ is a finitely generated projective R-module.

Proof. By the above remark it suffices to show that the map $\mu: J^{*} \otimes_{R} J \rightarrow \operatorname{Hom}_{R}(J, J)$ is an epimorphism. Since $\operatorname{Hom}_{R}(J, J)$ is a simple ring, it is enough to show that $\operatorname{Im} \mu \neq 0$. Since $J^{2} \neq 0$ there exist elements $r, s$ in $J$ such that $r s \neq 0$. Let $i$ denote the natural embedding of $J$ into $R$, then $\mu(i \otimes s)(r)=i(r) \cdot s=r s \neq 0$, thus $\mu(i \otimes s) \neq 0$.

We say that $R$ is a semiprimary ring if its Jacobson radical $N$ is nilpotent, and the residue ring $R / N$ is a semisimple (Artinian) ring.

Lemma 2. Any left ideal I in a semiprimary ring $R$ is the direct sum of a projective ideal and of an ideal contained in the radical $N$.

Received by the editors June 27, 1966.

1 This paper is based on a part of the author's doctoral dissertation, written at Brandeis University under the direction of Professor Maurice Auslander. 
Proof. Since $R$ is assumed to be a semiprimary ring, then every set of mutually orthogonal idempotents in $R$ is finite. Furthermore, every ideal that is not contained in the radical contains an idempotent.

Obviously, we may assume that $N \neq 0$.

If $I$ is contained in the radical we are done. Otherwise $I$ contains an idempotent. Let $\left(e_{1} \cdots e_{k}\right)$ be a maximal set of mutually orthogonal idempotents in $I$. It follows that $I$ admits a direct sum decomposition $I=R e_{1} \oplus \cdots \oplus R e_{k} \oplus I_{k}$ where $I_{k}=I \cap R\left(1-e_{1} \cdots-e_{k}\right)$. It suffices to prove that $I_{k}$ is contained in the radical. But if $I_{k}$ is not contained in the radical then $I_{k}$ contains an idempotent, say $e_{\boldsymbol{k}+1}^{\prime}$. Set $e_{k+1}$ $=e_{\boldsymbol{k}+1}^{\prime}-e_{1} e_{\boldsymbol{k}+1}^{\prime}-\cdots-e_{k} e_{\boldsymbol{k}+1}^{\prime}$ then one verifies by straightforward computations that $\left(e_{1} \cdots e_{k+1}\right)$ is a set of mutually orthogonal idempotents in $I$. This contradicts the maximality assumption on $\left(e_{1} \cdots e_{k}\right)$. Therefore $I_{k}$ is contained in the radical. This completes the proof of the lemma.

Theorem 1. Let $R$ be an Artinian ring with radical $N \neq 0$. There exists a left ideal $I$ for which $I^{2}=0, I N=0,1 . p \cdot \operatorname{dim} I=1 . p \cdot \operatorname{dim} N$, and $\operatorname{Hom}_{R}(I, I)$ is a division ring.

Proof. Let $J$ be an ideal of minimal length for which 1.p.dim $J$ $=1$.p.dim $N$.

We claim that if $f$ is a nonzero homomorphism of $J$ into $R$ then $f$ is a monomorphism. If this is not the case, then $\operatorname{ker} f$ and $\operatorname{Im} f$ are nonzero ideals in $R$. From the exact sequence $0 \rightarrow \operatorname{ker} f \rightarrow J \rightarrow \operatorname{Im} f \rightarrow 0$ it follows that $l(\operatorname{ker} f)<l(J)$ and that $l(\operatorname{Im} f)<l(J)$. Since gl.dim $R-1$ $=1$.p.dim $N[1]$, the minimality of $J$ implies that 1.p.dim $(\operatorname{ker} f)$ $<$ l.p.dim $N$, and that 1.p.dim $(\operatorname{Im} f)<1$.p.dim $N$. This leads to a contradiction since 1.p.dim $J \leqq \max \{1$.p.dim $(\operatorname{ker} f), 1 . p . \operatorname{dim}(\operatorname{Im} f)\}$.

In particular if $g$ is a nonzero homomorphism of $J$ into $J$ there results an exact sequence $0 \rightarrow J \rightarrow J \rightarrow J /$ Im $g \rightarrow 0$. By length argument it follows that $J / \operatorname{Im} g=0$. Thus $g$ is an isomorphism. Therefore $\operatorname{Hom}_{R}(J, J)$ is a division ring.

If $J N \neq 0$, then there exists an element $n, n \in N$, such that $J n \neq 0$. The map $j \rightarrow j n$ is a nonzero homomorphism of $J$ into $R$, thus $J n$ is isomorphic to $J$. Since $N$ is a nilpotent ideal one can proceed in that way to obtain an ideal $I$, isomorphic to $J$, and such that $I N=0$.

Finally we have to prove that $I^{2}=0$. If gl.dim $R>1$ this is immediate by Lemmas 1 and 2 since $\operatorname{Hom}_{R}(I, I)$ is a division ring. In case gl.dim $R=1$ we choose $I$ to be a minimal ideal in the radical for which $I N=0$. Obviously $\operatorname{Hom}_{R}(I, I)$ is a division ring and this ideal is of square zero. This completes the proof of the theorem. 
Corollary 1. Let $R$ be an Artinian ring with radical $N \neq 0$. There exists a two-sided ideal $K$ for which $K^{2}=0, K N=0$, and 1.p.dim $K$ $=1 . p \cdot \operatorname{dim} N$.

Proof. As in the proof of Theorem 1, we can find a left ideal $I$ in $R$ for which $I^{2}=0, I N=0$, l.p.dim $I=1 . p \cdot \operatorname{dim} N$, and every nonzero homomorphism of $I$ into $R$ is a monomorphism. Set $K=I R$. Then $K$ is a two-sided ideal in $R, K^{2}=(I R)(I R) \subset I^{2} R=0$, and $K N=(I R) N$ $C I N=0$. If there exists an element $r$ in $R$ such that $K=I r$, then $K$ is isomorphic to $I$ and we are done. Otherwise, there is a minimal set of elements $r_{1} \cdots r_{k}$ in $R$ such that $K=I r_{1}+\cdots+I r_{k}$. Set $I_{1}=I r_{1}$, and $I_{2}=I r_{2}+\cdots+I r_{k}$, then $I_{1}$ is isomorphic to $I$ and $I_{1} \cap I_{2} \neq I_{1}$. There results an exact sequence,

$$
0 \rightarrow I_{1} \cap I_{2} \rightarrow I_{1} \oplus I_{2} \rightarrow K \rightarrow 0 .
$$

Since $l\left(I_{1} \cap I_{2}\right)<l\left(I_{1}\right)$ it follows that 1.p.dim $\left(I_{1} \cap I_{2}\right)<$ l.p.dim $I_{1}$, thus 1.p.dim $N=1 . p \cdot d i m I_{1} \oplus I_{2} \leqq \max \left\{1 . p \cdot \operatorname{dim}\left(I_{1} \cap I_{2}\right), 1 . p \cdot \operatorname{dim} K\right\}$ $\leqq 1 . p \cdot \operatorname{dim} N$. Therefore 1.p.dim $K=1$. p.dim $N$.

2. A bound for the global dimension of an Artinian ring. Let $R$ be an Artinian ring with radical $N \neq 0$. Let $N=N_{1} \oplus \cdots \oplus N_{k}$ be a direct sum decomposition of $N$, i.e. $N_{i}$ are indecomposable ideals for $i=1, \cdots, k$. If $N$ is not a projective ideal, we choose $N_{1}$ so that $N_{1}$ is not a projective ideal, and furthermore $l\left(N_{1}\right) \geqq l\left(N_{i}\right)$ for all $i, 1 \leqq i \leqq k$, whenever $N_{i}$ is not a projective ideal.

For an integer $i \geqq 0$ we denote by $[i / 2]$ the minimal integer $j$ such that $j \geqq i / 2$. We set $[i / 2]=0$ for $i<0$.

An ideal $K$ is cyclic, if it is generated as a left ideal by a single element.

THEOREM 2. Let $R$ be an Artinian ring with radical $N \neq 0$. If 1.p.dim $J \leqq t<\infty$ whenever $J$ is a cyclic ideal for which $J^{2}=0, J N=0$, and $\operatorname{Hom}_{R}(J, J)$ is a division ring, then

$$
\text { gl.dim } R \leqq t+1+\left[\left(l\left(N_{1}\right)-2\right) / 2\right] .
$$

Proof. We prove by induction on the length of ideals that for every ideal $I$ in $R$ the following inequality holds:

$$
\text { 1.p.dim } I \leqq t+[(l(I)-2) / 2] \text {. }
$$

If $l(I)=1$ then $I$ is a minimal ideal, thus cyclic. Unless $I$ is a projective ideal it follows immediately that $I$ is isomorphic to a cyclic ideal $J$ for which $J^{2}=0, J N=0$, and $\operatorname{Hom}_{R}(J, J)$ is a division ring. Therefore 1.p.dim $I \leqq t$. 
If $l(I)=2$ we observe the following two cases:

Case 1. There exists a non zero homomorphism $f$ from $I$ into $R$ which is not a monomorphism. Therefore, $l(\operatorname{Im} f)=l(\operatorname{ker} f)=1$, and from the exact sequence $0 \rightarrow \operatorname{ker} f \rightarrow I \rightarrow \operatorname{Im} f \rightarrow 0$ it follows that 1.p.dim $I \leqq t$.

Case 2. Every nonzero homomorphism of $I$ into $R$ is a monomorphism. This necessarily implies that $I$ is a cyclic ideal and $\operatorname{Hom}_{R}(I, I)$ is a division ring. Unless $I$ is a projective ideal it follows that $I$ is isomorphic to a cyclic ideal $J$ for which $J^{2}=0$ and $J N=0$. Therefore 1.p.dim $I \leqq t$.

Assume that 1.p.dim $K \leqq t+[(l(K)-2) / 2]$ whenever $l(K)<n$. We may assume that $n \geqq 3$. Let $I$ be an ideal of length $n$. We again observe two cases:

Case 1. There exists a nonzero homomorphism of $I$ into $R$ which is not a monomorphism. This implies that $l(\operatorname{ker} f)<n$, and $l(\operatorname{Im} f)<n$. From the exact sequence $0 \rightarrow \operatorname{ker} f \rightarrow I \rightarrow \operatorname{Im} f \rightarrow 0$, it follows that:

l.p.dim $I \leqq \max \{1 \cdot \operatorname{p.dim}(\operatorname{ker} f), \operatorname{l} \cdot \operatorname{p.dim}(\operatorname{Im} f)\}$

$$
\begin{aligned}
& \leqq t+\max \{[(l(\operatorname{ker} f)-2) / 2],[(l(\operatorname{Im} f)-2) / 2]\} \\
& \leqq t+(l(I)-2) / 2] .
\end{aligned}
$$

Case 2: Every nonzero homomorphism of $I$ into $R$ is a monomorphism. Unless $I$ is a projective ideal, there exists an ideal $J$ isomorphic to $I$ for which $J^{2}=0, J N=0$, and $\operatorname{Hom}_{R}(J, J)$ is a division ring. If $J$ is a cyclic ideal then 1.p.dim $I \leqq t$. Otherwise there exists a minimal set of elements $i_{1} \cdots i_{k}$ in $I$ such that $I=R i_{1}+\cdots+R i_{k}$. Set $I_{1}=R i_{1}$, and $I_{2}=R i_{2}+\cdots+R i_{k}$ then $I_{1} \cap I_{2} \neq I_{2}$, and $I_{1} \cap I_{2} \neq I_{1}$. From the exact sequence $0 \rightarrow I_{1} \cap I_{2} \rightarrow I_{1} \oplus I_{2} \rightarrow I \rightarrow 0$ it follows that:

l.p.dim $I \leqq \max \left\{1 . p \cdot \operatorname{dim}\left(I_{1} \cap I_{2}\right)+1\right.$, l.p.dim $I_{1}$, l.p.dim $\left.I_{2}\right\}$

$$
\begin{aligned}
& \leqq t+\max \left\{\left[\frac{l\left(I_{1} \cap I_{2}\right)-2}{2}\right]+1,\left[\frac{l\left(I_{1}\right)-2}{2}\right],\left[\frac{l\left(I_{2}\right)-2}{2}\right]\right\} \\
& \leqq t+\left[\frac{l(I)-2}{2}\right]
\end{aligned}
$$

since $l(I)-2 \geqq \max \left\{l\left(I_{1} \cap I_{2}\right), l\left(I_{1}\right)-2, l\left(I_{2}\right)-2\right\}$, and since from $n \geqq 3$ it follows that $[(l(I)-2) / 2] \geqq 1$.

We conclude the proof by observing that if $N$ is projective then gl. $\operatorname{dim} R=1$. Otherwise an ideal $N_{1}$ exists as above and we have:

$$
\begin{array}{r}
\text { l.p.dim } N_{i} \leqq t+\left[\left(l\left(N_{i}\right)-2\right) / 2\right] \leqq t+\left[\left(l\left(N_{1}\right)-2\right) / 2\right] \\
\quad \text { for } i=1, \cdots, k
\end{array}
$$


therefore gl.dim $R=1 . p . \operatorname{dim} N+1 \leqq t+1+\left[\left(l\left(N_{1}\right)-2\right) / 2\right]$.

\section{REFERENCES}

1. M. Auslander, On the dimension of modules and algebras. III. Global dimension, Nagoya Math. J. 9 (1955), 67-77.

2. M. Auslander and O. Goldman, Maximal orders, Trans. Amer. Math. Soc. 97 (1960), 1-24.

3. H. Cartan and S. Eilenberg, Homological algebra, Princeton Univ. Press, Princeton, N. J., 1956.

4. N. Jacobson, Structure of rings, Colloq. Publ., Vol. 37, Amer. Math. Soc., Providence, R. I., 1956.

BRANDEIS UNIVERSITY AND

TEChNion, Haifa 